\title{
TECNOLOGÍAS DE LA INFORMACIÓN Y LA COMUNICACIÓN EN LA EDUCACIÓN SUPERIOR: UNA REVISIÓN DOCUMENTAL
}

\section{INFORMATION AND COMMUNICATION TECHNOLOGIES IN HIGHER EDUCATION: A DOCUMENTARY ANALYSIS}

\author{
Adriana Rangel Baca \\ adrianna.rangel@hotmail.com \\ Escuela Superior de Comercio y Administración, Unidad Santo Tomás. \\ Sección de Estudios de Posgrado e Investigación. Departamento de Posgrado. \\ Prolongación Manuel Carpio 471, Col. Plutarco Elías Calles, Del. Miguel Hidalgo, \\ 11340, \\ México, Distrito Federal.
}

Resumen: El objetivo de este trabajo es presentar el estado de la investigación académica sobre TIC en educación superior durante el periodo 2005-2015, a través del análisis de artículos divulgados en publicaciones incluidas en el Índice de Revistas Mexicanas de Investigación Científica y Tecnológica del Consejo Nacional de Ciencia y Tecnología (Conacyt). Para la búsqueda y selección de los artículos potencialmente relevantes se definieron unos criterios de inclusión y exclusión y se diseñó una ficha de recolección para facilitar su registro y análisis. Los resultados muestran que las publicaciones sobre TIC en educación superior no han crecido al mismo ritmo que las experiencias que se han venido realizando en los últimos años y que prevalecen los estudios de tipo descriptivo o exploratorio lo que dificulta la generalización de los hallazgos reportados.

Palabras clave: Análisis documental, Educación superior, Tecnologías de la información y comunicación

Abstract: The purpose of this paper is to present the State of academic research on ICT in higher education during the period 2005-2015 through the analysis of items reported in publications inclunding in the Index of Mexican Journals of Scientific and Technological Research of the National Council of Science and Technology (Conacyt). Inclusion and exclusion criteria were defined for the search and selection of potentially relevant articles and a collection form was designed to facilitate its registration and analysis.

The results show that the publications on ICT in higher education have not grown at the same rate as the experiences that have been made in recent years, and type descriptive or exploratory studies prevail which makes the generalize the reported findings.

Keywords: Documentary analysis, Higher education, Information and communications technology (ICT)

$$
-125-
$$

Píxel-Bit. Revista de Medios y Educación. No 52 Enero 2018. ISSN: 1133-8482. e-ISSN: 2171-7966. doi: http://dx.doi.org/10.12795/pixelbit.2018.i52.09 


\section{Introducción.}

La investigación en TIC ha pasado por diversas etapas en las que se han producido cambios tanto en los problemas de investigación planteados, como en la metodología utilizada. Las primeras investigaciones de las que se tiene conocimiento están relacionadas con el estudio de los medios como antecedentes de las TIC, y datan de 1918. Sin embargo, se considera que es hasta la década de los cincuenta cuando este tipo de estudios comienza a desarrollarse de forma más sistemática (Vidal, 2006, p. 540).

En la década de los sesenta, el auge de los medios de comunicación de masas fue un hecho que sin duda influyó en el desarrollo de la investigación en TIC, debido básicamente a las posibilidades de uso y a las ventajas que representaba la introducción de la radio y la televisión en la educación (Vidal, 2006, p. 540).

En los años setenta, el desarrollo de la informática permitió que se consolidara la utilización de los ordenadores con fines educativos, concretamente en aplicaciones como la Enseñanza Asistida por Ordenador (EAO) (Vidal, 2006, p. 241). Por lo tanto, los estudios realizados durante esta época se concentraron en el análisis de dos aspectos: a) la distribución y el uso de las computadoras en las escuelas y b) el impacto del uso de las computadoras en el rendimiento académico (Area, 2005, p. 3).

A mediados de la década de los ochenta, se introducen en la educación materiales electrónicos innovadores que hacen evidente que los efectos de las TIC sobre la enseñanza y el aprendizaje podrían ser comprendidos solo si se analizaban como parte de la interacción de múltiples factores en el mundo complejo de las escuelas (Area, 2005, p. 4).

Finalmente, y como consecuencia de la etapa anterior, en la década de los noventa los esfuerzos se concentraron en sistematizar o identificar el estado de la cuestión sobre los factores y procesos de integración y uso escolar de las TIC (Area, 2005, p. 4), porque a pesar de que se tenían evidencias empíricas sobre el uso de estas herramientas, no se contaba con un cuerpo teórico que permitiera explicar «el conjunto de fenómenos y factores asociados no sólo con la generalización de las TIC a gran escala en los sistemas escolares, sino también que [explicara o conceptualizara] cómo se generan procesos de innovación y mejora educativa trabajando con ordenadores en los centros y aulas» (Area, 2005, p. 4).

En México, se han publicado algunos trabajos bajo esta línea de investigación (Alarcón y Ortiz, 2014; Amador, 2003 y Ramírez, 2001), sin embargo, aún en la actualidad se carece de un cuerpo teórico que permita, entre otros aspectos: 1) identificar los factores que favorecen la integración de estas herramientas en el aula, 2) conocer el impacto del uso de la tecnología en los resultados educativos y 3 ) determinar las mejores prácticas en los procesos de integración escolar de las TIC. Por ello se planteó el presente estudio, el cual pretende contribuir a la sistematización del estado de la investigación académica sobre TIC en la educación superior, mediante el análisis de las publicaciones que se han desarrollado en los últimos 10 años en cinco revistas especializadas, a saber: Perfiles Educativos, Revista de la Educación Superior, Revista Electrónica de Investigación Educativa, Revista Mexicana de Investigación Educativa y Sinéctica.

$$
-126-
$$




\section{Método}

\subsection{Tipo de investigación}

Se trata de una investigación de tipo documental en un nivel descriptivo, ya que comporta la revisión minuciosa de los diferentes artículos recuperados, y trabajo de campo que se realiza con el fin de dar cuenta de los diferentes estudios que se han realizado, de los objetos de estudio, de las delimitaciones espaciales, temporales y contextuales, así como de las metodologías y resultados obtenidos (Hoyos, 2000).

\subsection{Unidad de análisis}

La unidad de análisis quedó comprendida por 52 artículos de investigación, previamente validados.

\subsection{Instrumento}

Se construyó una ficha de recolección en Excel, cuyas categorías de análisis quedaron organizadas en dos grupos de datos:

1. Datos descriptivos: Título, año de publicación, fuente, autor/es, institución de procedencia, área de conocimiento.

2. Datos relevantes: Objetivo, tema, tipo de estudio, sujetos de la investigación, enfoque, diseño metodológico, técnicas de investigación y resultados.

\section{Procedimiento}

Una vez delimitado el tema de investigación, se estableció como condición previa al proceso de búsqueda y recopilación de las publicaciones, que las fuentes primarias de información serían las revistas de

\begin{tabular}{ll}
\hline \multicolumn{1}{c}{ Criterio } & \multicolumn{1}{c}{ Valor } \\
\hline Autor/es & Investigador/es de nacionalidad mexicana \\
Año & Comprendido entre el periodo 2005-2015 \\
Tipo de publicación & Artículo de investigación \\
Metodología y diseño & El mismo que aparece en el manuscrito \\
Objetivo del estudio & El mismo que aparece en el manuscrito \\
Participantes & Los mismos que aparecen en el manuscrito \\
Resultados & Los mismos que aparecen en el manuscrito \\
Idioma & Español \\
\hline
\end{tabular}

Tabla 1: Criterios de inclusión y exclusión de fuentes primarias. Fuente: Elaboración propia

-127 -

Píxel-Bit. Revista de Medios y Educación. $N^{o} 52$ Enero 2018. ISSN: 1133-8482. e-ISSN: 2171-7966. doi: http://dx.doi.org/10.12795/pixelbit.2018.i52.09 


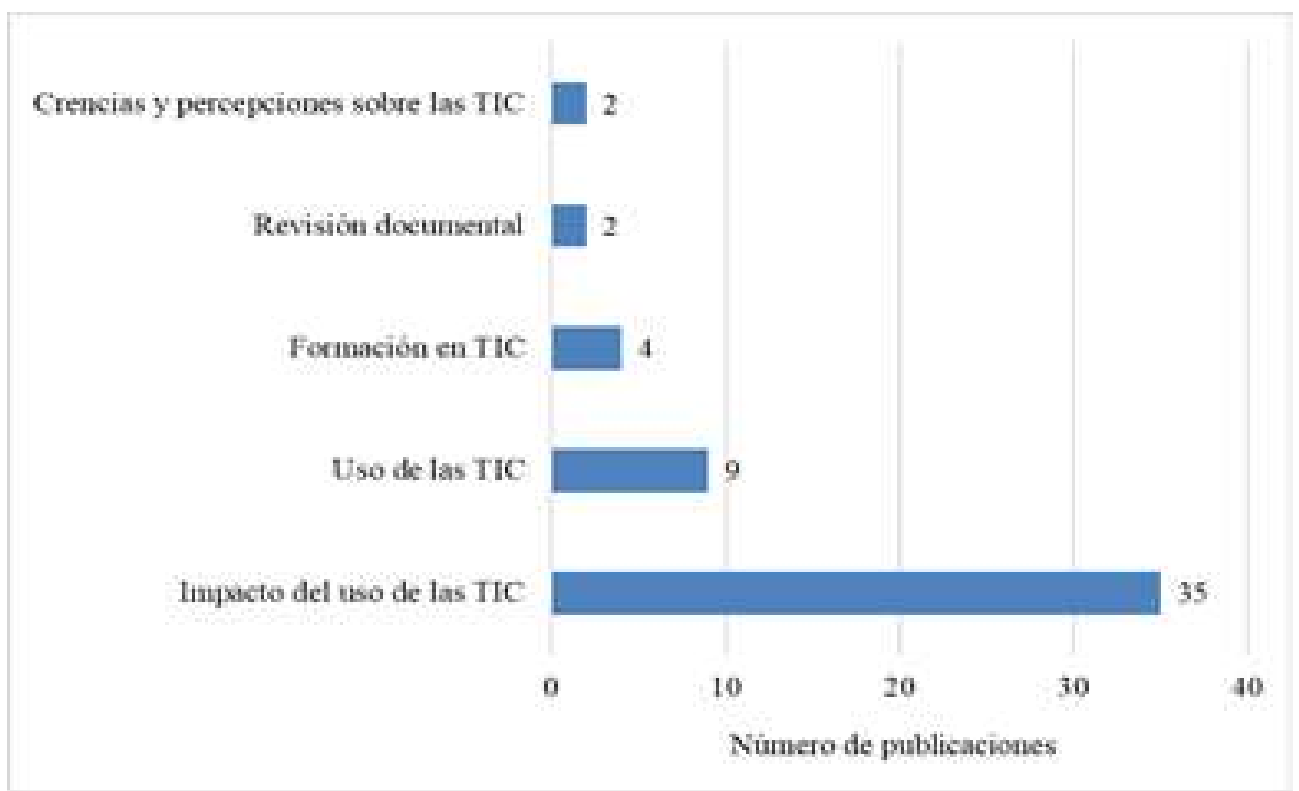

Figura 1. Ejes temáticos hallados en el campo de estudio. Fuente: Elaboración propia.

educación superior incluidas en el Índice de Revistas Mexicanas de Investigación Científica y Tecnológica del Conacyt, que tuvieran acceso vía Internet a todos los números del periodo comprendido entre el 2005 y el 2015. Para la búsqueda, recuperación y análisis de los manuscritos, se siguieron una serie de fases: a) definición de los criterios de exclusión e inclusión de los manuscritos (Ver Tabla 1), b) búsqueda y localización de los artículos potencialmente relevantes, c) acceso a los textos para su lectura, revisión y copiado textual de los datos, según las categorías de análisis definidas en la ficha de recolección y d) análisis de datos, el cual incluyó dos tipos de lecturas: una lineal, para lograr la comprensión del texto e identificar ideas clave; y otra transversal, para identificar consensos, singularidades, desacuerdos o vacíos entre las diferentes fuentes analizadas.

\section{Resultados}

\subsection{Principales temas en el campo de} estudio

En general, el interés de los académicos se centra en el estudio del impacto de las TIC en los procesos de enseñanza aprendizaje (67.31\%). Particularmente, les interesa conocer si existe una relación significativa entre el uso de estas herramientas y el desempeño académico de los alumnos (64.51\%), el desempeño profesional de los docentes $(22.58 \%)$ o el desempeño de ambos actores $(12.90 \%)$.

El segundo tema más estudiado es el uso de las TIC (17.31\%). De acuerdo con la muestra recuperada, el $81.81 \%$ de los académicos busca identificar para qué usan las TIC los alumnos, mientras que el 18.18\%, pretende conocer el uso que hacen de estas herramientas los profesores.

$$
-128-
$$


Los temas que resultan de menor interés entre la comunidad académica son: formación en TIC $(7.69 \%)$, revisiones documentales $(3.85 \%)$ y creencias y percepciones sobre el uso de las TIC (3.85\%).

\subsection{Evolución cronológica de las investigaciones}

Con respecto a la evolución cronológica de las investigaciones, se observa que la producción académica en este campo de estudio no muestra un crecimiento constante. De hecho, en algunos años ésta es prácticamente inexistente (2009 y 2011) y en algunos otros, solo alcanza una publicación por año $(1.92 \%, 2005$ y 2007).

A partir del 2012 dicha situación se revierte, ya que se reportan 8 publicaciones para ese año (15.38\%). Sin embargo, su número sigue siendo limitado y su crecimiento bastante intermitente con una tendencia más bien a la baja.

\subsection{Institución de procedencia de los autores}

De acuerdo con los resultados, el 28.84\% de los autores y coautores de los artículos de investigación seleccionados está adscrito a la Universidad Nacional Autónoma de México (UNAM, máxima casa de estudios de México), mientras que el $53.84 \%$ proviene de académicos adscritos a alguna universidad estatal o instituto tecnológico, tales como el Instituto Tecnológico de Estudios Superiores de Monterrey (ITESM, 19.23\%), la Universidad Autónoma de Baja California (9.62\%), la Universidad Veracruzana (5.77\%) o la Universidad Autónoma del Estado de Morelos (5.77\%).

Es importante mencionar que la producción académica se genera principalmente en el centro $(59.62 \%)$ y norte $(40.38 \%)$ del país, lo que hace evidente la escasa proliferación de este tipo de publicaciones en el sur del territorio mexicano.

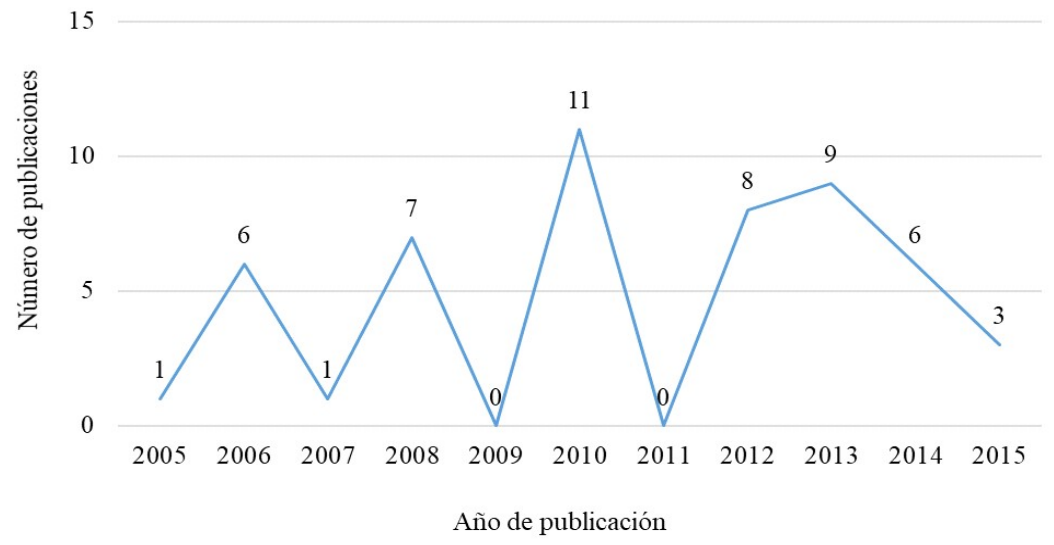

Figura 2. Distribución de artículos por año de publicación. Fuente: Elaboración propia.

- 129 -

Píxel-Bit. Revista de Medios y Educación. No 52 Enero 2018. ISSN: 1133-8482. e-ISSN: 2171-7966. doi: http://dx.doi.org/10.12795/pixelbit.2018.i52.09 


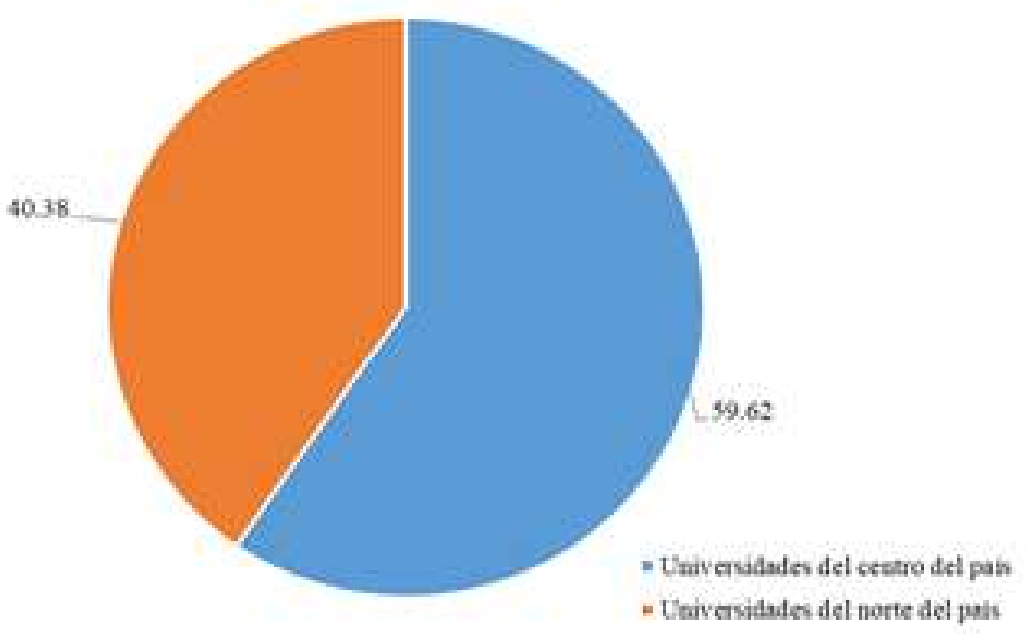

Figura 3. Distribución de publicaciones por área geográfica. Fuente: Elaboración propia.

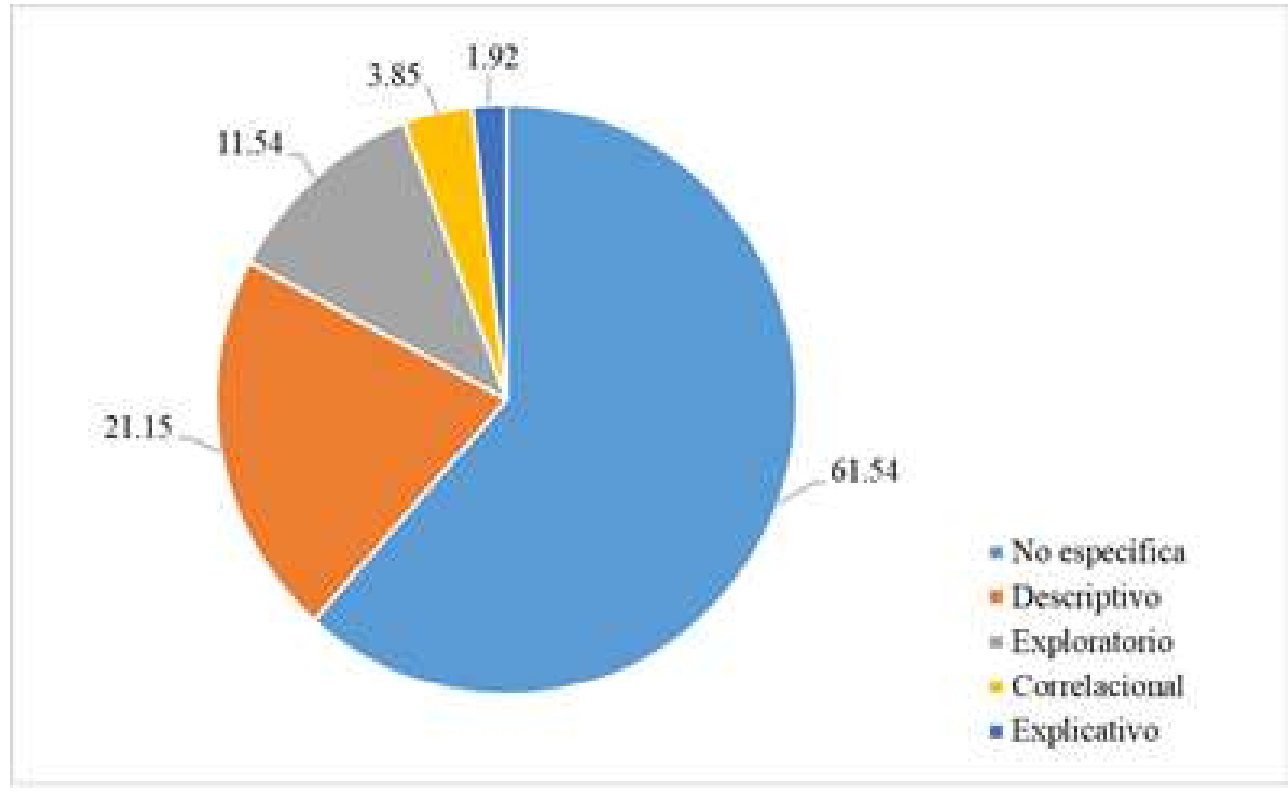

Figura 4. Distribución de publicaciones por tipo de estudio. Fuente: Elaboración propia.

$-130-$

Píxel-Bit. Revista de Medios y Educación. No 52 Enero 2018. ISSN: 1133-8482. e-ISSN: 2171-7966. doi: http://dx.doi.org/10.12795/pixelbit.2018.i52.09 


\subsection{Tipo de estudio}

En cuanto al tipo de estudio, se observa que el universo de publicaciones se divide en dos grandes grupos: los que expresan explícitamente de qué tipo de estudio se trata (38.46\%) y los que no lo hacen (61.54\%). Dentro del primer grupo la distribución de los trabajos fue la siguiente: $21.15 \%$ eran estudios de carácter descriptivo; $11.54 \%$, exploratorio; $3.85 \%$, correlacional y $1.92 \%$, explicativo. Aunque no se cuenta con información explícita es posible inferir que el segundo grupo de trabajos son mayoritariamente estudios de tipo descriptivo, ya que buscan identificar propiedades, características o perfiles de los actores educativos involucrados en entornos mediados por la tecnología.
En cuanto a la forma como se recupera la información, se tiene que el $25 \%$ de los autores utiliza el enfoque mixto; el $21.15 \%$, cuantitativo; el $8 \%$, cualitativo y el porcentaje restante $(20 \%)$, no lo específica. Ahora bien, entre los instrumentos o técnicas más utilizadas para la recolección de datos fueron el cuestionario (23.08\%), el cuestionario en combinación con otros instrumentos $(19.23 \%)$ y las entrevistas, también en combinación con otros instrumentos (17.31\%). En menor medida se recurrió a herramientas de tipo tecnológico (9.62\%) o instrumentos de evaluación (5.77\%).

Como puede observarse, la necesidad de afrontar la complejidad de los problemas de investigación planteados en este campo de estudio ha llevado a los autores a recurrir, cada vez más, al enfoque mixto y a la combinación de diferentes técnicas de estudio

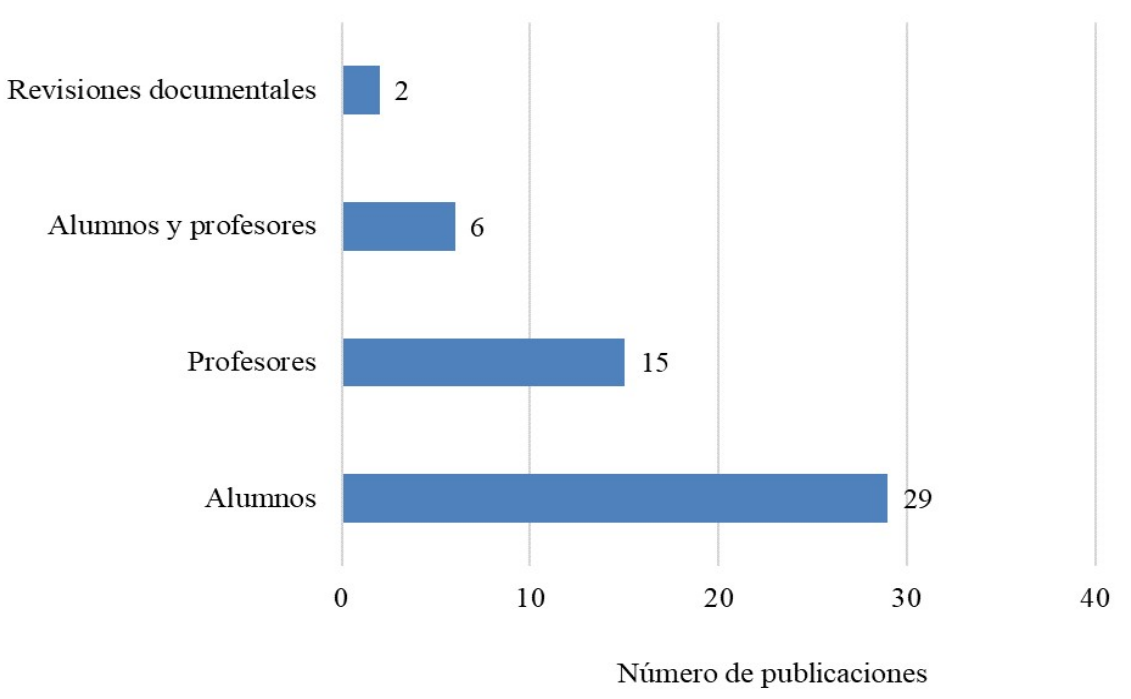

Figura 5. Distribución de publicaciones por tipo de sujeto de la investigación. Fuente: Elaboración propia.

$-131-$

Píxel-Bit. Revista de Medios y Educación. No 52 Enero 2018. ISSN: 1133-8482. e-ISSN: 2171-7966. doi: http://dx.doi.org/10.12795/pixelbit.2018.i52.09 
para obtener resultados de orden superior que permitan profundizar en el conocimiento de la realidad estudiada.

\subsection{Sujetos de la investigación}

En el conjunto de publicaciones los sujetos más estudiados son los alumnos (55.77\%), particularmente, los alumnos de educación superior $(86.21 \%)$, del nivel medio superior $(10.34 \%)$ y de la educación para adultos (3.45\%). Por orden de importancia, le siguen los profesores, cuyas publicaciones representan el $28.85 \%$, de donde el $73.33 \%$ corresponde a profesores del nivel superior, el $13.33 \%$ a profesores del nivel medio superior y un porcentaje similar, a profesores de educación básica. Solo el $11.54 \%$ se concentra en el estudio de ambos actores (alumnos y profesores), mientras que el porcentaje restante $(3.85 \%)$ son estudios de tipo documental.

3.6. Consensos y singularidades de la investigación académica

Para presentar los resultados de la investigación académica se identificaron los principales consensos y particularidades a partir de la información recuperada.

\subsubsection{Consensos}

Por consensos se entiende a las ideas generalizadas entre las publicaciones que integran la muestra.

En este sentido, el primer consenso es que el uso de las TIC tiene un impacto positivo en el aprendizaje de los estudiantes (Benítez, Barajas \& Hernández, 2014; García \& Pineda, 2010; Hernández, Sánchez, Rodríguez, Caballero \& Martínez, 2014; Inzunsa, 2010;
Peñalosa \& Castañeda, 2008; Torreblanca \& Rojas, 2010; Úrzua \& López, 2010). El uso de la tecnología no solo contribuye a la generación de conocimiento, sino que también diversifica los medios para hacerlo, lo cual evidentemente potencializa el aprendizaje. La posibilidad de interacción que ofrecen los distintos dispositivos tecnológicos es otro aspecto que lo favorece, ya sea de manera individual o colectivamente (Torres y Jaimes, 2015, p. 1).

El segundo consenso es que el uso de las TIC favorece el desarrollo de habilidades de orden superior, tales como:

a) la capacidad de decidir y comunicar y de reflexionar sobre la pertinencia del contenido y sobre la propia manera de aprender (Morán, 2010, p. 126);

b) la capacidad de mejorar la búsqueda de información, la reflexión crítica y el posicionamiento personal y de generar una mayor capacidad autocrítica en los alumnos, particularmente, sobre su dominio de la lengua escrita (Hernández, Sánchez, Rodríguez, Caballero \& Martínez, 2014; Monzón, 2011; Reyes, Fernández \& Martínez, 2013); y

c) la capacidad de reflexionar sobre la propia identidad profesional y sobre la trayectoria personal y académica (Díaz Barriga, Romero \& Heredia, 2012, p. 9).

Finalmente, el tercer consenso es que las TIC son un instrumento valioso como método de evaluación, ya que no solo permiten dar seguimiento a la participación o actuación de los estudiantes (Díaz Barriga, Romero \& Heredia, 2012; Hernández, Sánchez, Rodríguez, Caballero \& Martínez, 2014; Reyes, Fernández \& Martínez, 2013), sino que además resultan ser una herramienta efectiva para la autoevaluación del desempeño docente (Arbesú \& Gutiérrez, 2014, p. 106).

-132 -

Píxel-Bit. Revista de Medios y Educación. No 52 Enero 2018. ISSN: 1133-8482. e-ISSN: 2171-7966. doi: http://dx.doi.org/10.12795/pixelbit.2018.i52.09 


\subsubsection{Singularidades}

Las singularidades, en cambio, son aquellos resultados que si bien no pueden ser generalizables destacan por su originalidad.

El primero de ellos sostiene que el éxito de los estudiantes en un entorno educativo mediado por la tecnología depende fundamentalmente de su perfil académico, así como de las habilidades de autogestión que posea (Martínez \& Heredia, 2010, p. 371). Aspectos de tipo sociodemográfico, tales como edad, sexo, escuela de procedencia o área de conocimiento no reportan alguna influencia significativa en los resultados académicos alcanzados (Moreno y Cárdenas, 2012, p. 135; Peñalosa, Landa y Castañeda, 2010, p. 453).

Otro estudio señala que las TIC pueden integrarse a los procesos de aprendizaje y a las organizaciones comunitarias sin requerir de cursos previos de cómputo, ya que los jóvenes y adultos del medio rural utilizan la tecnología de manera casi inmediata, siempre y cuando su uso esté vinculado con el desarrollo de pequeños proyectos que sean de su interés. En este contexto las TIC fueron utilizadas para apoyar la organización interna, para recuperar tradiciones comunitarias $y$, específicamente, para comunicar sentimientos, valores e ideas en el ámbito local (Salinas, Huerta, Porras, Amador \& Ramos, 2006, p. 58).

Los resultados de otro estudio revelan que el uso de las TIC no es en sí mismo un elemento que lleve a los profesores a cuestionar los fundamentos de su práctica docente, sin embargo, si es posible que su uso genere en ellos la necesidad de cuestionar su rol actual en sus relaciones con el saber, con el control, así como, con la forma en que se relacionan con los estudiantes (Juárez, Buenfil \& Trigueros, 2008, p. 1081).

Por otro lado, también se concluye que la disponibilidad de infraestructura tecnológica no es un detonante de la calidad y equidad educativa (Cano \& Vaca, 2014, p. 9) ni tampoco implica que se haya logrado una adecuada apropiación y uso de las mismas para mejorar los procesos de enseñanza aprendizaje (Torres, Barona \& García, 2010, p. 105).

Finalmente, destaca un estudio que busca estimar las horas/aula que se liberarían si el conocimiento explícito se delegara a tecnologías y el aula se usara para transmitir el conocimiento tácito. Los resultados indican que es posible aumentar hasta en $60 \%$ la capacidad de aulas o en $120 \%$ la atención a los estudiantes, por lo tanto, los autores sugieren que el tiempo liberado podría usarse para seguimiento, tutorías y diseño de experiencias de aprendizaje (McAnally, Navarro \& Rodríguez, 2006, p. 11).

\section{Discusión}

Con base en la revisión documental, se tienen las siguientes precisiones sobre el análisis de este conjunto de publicaciones:

$\mathrm{Si}$ bien es cierto que las publicaciones académicas sobre TIC han ido en aumento, su crecimiento no ha sido constante ni tampoco representativo, ya que el número de estudios realizados sobre este fenómeno no corresponde con el desarrollo alcanzado por estas herramientas en los últimos años (Alarcón \& Ortiz, 2014; Amador, 2003 \& Ramírez, 2001).

Del mismo modo, se observa que los resultados de los estudios revisados siguen sin poder ser generalizables, ya que la mayoría de ellos son trabajos de tipo

$-133-$

Píxel-Bit. Revista de Medios y Educación. No 52 Enero 2018. ISSN: 1133-8482. PIXEL e-ISSN: 2171-7966. doi: http://dx.doi.org/10.12795/pixelbit.2018.i52.09 
descriptivo o exploratorio que presentan experiencias sobre el uso que se hace de las TIC (Inzunsa, 2010; McAnally, Navarro \& Rodríguez, 2006; Moreno \& Cárdenas, 2012) o sobre los efectos que se generan cuando son utilizadas con fines educativos, en un contexto específico y en un momento determinado (Cuevas, García \& Cruz, 2008; Díaz Barriga, Romero \& Heredia, 2012; Torreblanca y Rojas, 2010; Urzúa \& López, 2010). En ningún caso se reportan estudios a nivel nacional o de corte longitudinal que permitan dar mayor validez y confiabilidad a los resultados obtenidos (Alarcón \& Ortiz, 2014; Amador, 2003 \& Ramírez, 2001).

Por otro lado, se observa un cambio sustancial en los temas de interés. El acceso, la disponibilidad y el uso que se hace de las TIC, así como las percepciones de los actores educativos ante el uso de estas herramientas, son temas cada vez menos estudiados. Lo que interesa hoy por hoy a los investigadores es poder dilucidar el impacto que estas herramientas generan en el desempeño académico de los estudiantes (Alarcón \& Ortiz, 2014) y muestran poco interés por conocer y analizar los fenómenos socioculturales y curriculares presentes en algunas experiencias donde se ha logrado integrar con éxito la tecnología (Area, 2005, p. 17).

Otro aspecto que vale la pena señalar es la desconcentración que ha sufrido la producción académica a lo largo del periodo analizado. En los primeros años, la UNAM, junto con algunas universidades del centro del país, concentraban el mayor número de publicaciones en este campo de estudio. Sin embargo, en los últimos cinco años, las instituciones del norte de la República Mexicana son quienes más artículos han reportado (Farías, Pedraza \& Lavín, 2013;
García, Corrales \& Maldonado, 2013; Inzunsa, 2010; McAnally, Navarro \& Rodríguez, 2006). Se observa entonces que las aplicaciones y experiencias en TIC se han ido multiplicando casi de manera exclusiva en algunos estados, haciendo evidente la brecha existente entre el centro-norte y el sur del país en materia de infraestructura, recursos y conocimiento. Dicha situación exige centrar el análisis en aquellos estados donde es escasa la producción académica sobre este campo de estudio, a fin de dilucidar las razones de esta supuesta escasez o, en su caso, conocer cuál ha sido su experiencia en el uso de estas herramientas.

Asimismo, vale la pena mencionar que cada vez son más los trabajos que se elaboran de manera colaborativa, ya sea interinstitucional o interdisciplinariamente, debido a que la complejidad del fenómeno exige analizarlo desde distintos enfoques, pero también a que cada vez son más los profesores interesados en aprovechar las potencialidades que estas herramientas les ofrecen.

\section{Referencias bibliográficas}

Alarcón, E. y Ortiz, V. (2014). Estudiantes, profesores y TIC. La investigación en México. En A. Ramírez y M. A. Casillas (Ed.). Háblame de TIC. Tecnología Digital en la Educación Superior (pp. 39-70). Argentina: Brujas.

Amador Bautista, R. (2003). Valoración final de la investigación. En A. D. López (Coord.), Saberes Científicos, Humanísticos y Tecnológicos: procesos de enseñanza y aprendizaje. Tomo II. (331-341). México: COMIE.

Arbesú García, M. I. y Gutiérrez Martínez, E. (2014). El portafolios formativo. Un recurso para la reflexión y auto-evaluación en la docencia. Perfiles Educativos, 36(143).

- 134 -

Píxel-Bit. Revista de Medios y Educación. No 52 Enero 2018. ISSN: 1133-8482. e-ISSN: 2171-7966. doi: http://dx.doi.org/10.12795/pixelbit.2018.i52.09 
Recuperado de http://www.scielo.org.mx/ scielo.php?script=sci_arttext\&pid=S018526982014000100007

Area, M. (2005). Tecnología de la información y comunicación en el sistema escolar: una revisión de las líneas de investigación. Revista Electrónica de Investigación y Evaluación Educativa, 11. Recuperado de http://www.uv.es/relieve/ v11n1/RELIEVEv11n1_1.htm

Benítez Lima, M. G., Barajas Villarruel, J. I. y Hernández Uresti, I. N. (noviembre, 2014). Efecto de la aplicación de una estrategia de comprensión de lectura en un entorno virtual. Revista Electrónica de Investigación Educativa, 16(3). Recuperado de http:// w w w. s c i e 1 o . o r g . $\mathrm{m} \mathrm{x} \mathrm{/}$ scielo.php?script $=$ sci_arttext\&pid $=$ S160740412014000300005

Cano Ruiz, A. y Vaca Uribe, J. (2013). Usos iniciales y desusos de la estrategia «Habilidades digitales para todos» en escuelas secundarias de Veracruz. Perfiles Educativos, 35(142). Recuperado de http:// revistas.unam.mx/index.php/perfiles/article/ view/42572/38679

Cuevas Salazar, O., García López, R. I. y Cruz Medina, I. R. (octubre-diciembre, 2008). Evaluación del impacto de una plataforma para la gestión del aprendizaje utilizada en cursos presenciales en el Instituto Tecnológico de Sonora. Revista Mexicana de Investigación Educativa, 13(39). Recuperado de http://www.scielo.org.mx/pdf/rmie/v13n39/ v13n39a4.pdf

Díaz Barriga Arceo, F., Romero Martínez. E. y Heredia Sánchez, A. (2012). Diseño tecnopedagógico de portafolios electrónicos de aprendizaje: una experiencia con estudiantes universitarios. Revista Electrónica de Investigación Educativa,
14(2). Recuperado de http://redie.uabc.mx/ redie/article/view/313/670

Farías, G., Pedraza, N. y Lavín, J. (2013). Gestión de un programa de capacitación en línea para el desarrollo de habilidades y capacidades TIC en profesores de negocios. Revista Electrónica de Investigación Educativas, 15(1). Recuperado de http:// redie.uabc.mx/redie/article/view/329/512

García Alcaraz, J. L., Corrales Prieto, R. A. y Maldonado Macías, A. A. (2013). Uso de laptops por estudiantes universitarios y su impacto en la eficiencia académica. Revista Mexicana de Investigación Educativa, 18(57). Recuperado de http:// www.redalyc.org/pdf/140/14025774011.pdf

García Cabrero, B. y Pineda Ortega, V. J. (enero-marzo, 2010). La construcción de conocimiento en foros virtuales de discusión entre pares. Revista Mexicana de Investigación Educativa, 15(44). Recuperado de http://www.comie.org.mx/documentos/ rmie/v15/n044/pdf/ART44006.pdf

Hernández Rojas, G., Sánchez González, P., Rodríguez Varela, E. I., Caballero Becerril, R. S. y Martínez Martínez, M. (2014). Un entorno b-learning para la promoción de la escritura académica de estudiantes universitarios. Revista Mexicana de Investigación Educativa, 19(61). Recuperado de http:// w w w. s c i e 1 o . o r g . m x / scielo.php?script $=$ sci_arttext\&pid $=\mathrm{S} 1405$ 66662014000200002

Hoyos, C. (2000). Un modelo para Investigación Documental: Guía teóricopráctica sobre construcción de estados del arte. Medellín: Señal Editora.

Inzunsa Cazares, S. (abril-junio 2010). Entornos virtuales de aprendizaje: un enfoque alternativo para la enseñanza y aprendizaje de la inferencia estadística. Revista Mexicana de Investigación Educativa, 15(45).

- 135 -

Píxel-Bit. Revista de Medios y Educación. No 52 Enero 2018. ISSN: 1133-8482. e-ISSN: 2171-7966. doi: http://dx.doi.org/10.12795/pixelbit.2018.i52.09 
Recuperado de http://www.scielo.org.mx/ scielo.php?script $=$ sci_arttext\&pid $=\mathrm{S} 1405$ 66662010000200005

Juárez, M., Buenfil, R. N. y Trigueros, M. (octubre-diciembre 2008). De las prácticas convencionales a los ambientes de aprendizaje colaborativo a distancia: un estudio con profesores de ciencias de bachillerato desde la Teoría de la actividad. Revista Mexicana de Investigación Educativa 13(39). Recuperado de http:// w w w. s c i e 1 o. o r g . $\mathrm{m} \mathrm{x} /$ scielo.php?script $=$ sci_arttext\&pid $=\mathrm{S} 1405$ 66662008000400003

Martínez Martínez, R. y Heredia Escorza, Y. (abril-junio, 2010). Tecnología educativa en el salón de clase: estudio retrospectivo de su impacto en el desempeño académico de estudiantes universitarios del área de Informática. Revista Mexicana de Investigación Educativa, 15(45). Recuperado de http://www.scielo.org.mx/ scielo.php?script $=$ sci_arttext\&pid $=\mathrm{S} 1405$ 66662010000200003

McAnally Salas, L., Navarro Hernández, M. R. y Rodríguez Lares, J. J. (enero-marzo, 2006). La integración de la tecnología educativa como alternativa para ampliar la cobertura en la educación superior. Revista Mexicana de Investigación Educativa, 11(28). Recuperado de http://www.redalyc.org/ articulo.oa?id=14002803

Morán Oviedo, P. (enero, 2010). Aproximaciones teórico-metodológicas en torno al uso del portafolio como estrategia de evaluación del alumno en la práctica docente. Experiencia en un curso de Laboratorio de Didáctica en la docencia universitaria. Perfiles Educativos, 32(129). Recuperado de http://www.scielo.org.mx/ scielo.php?script $=$ sci_arttext\&pid $=$ S018526982010000300007
Monzón Laurencio, L. A. (2011). El blog y el desarrollo de habilidades de argumentación y trabajo colaborativo. Perfiles educativos, 33(131). Recuperado de http:// w w w. s c i e 1 o . o r g . m x / scielo.php?script $=$ sci_arttext\&pid $=\mathrm{S} 0185$ 26982011000100006

Moreno Almazán, O. y Cárdenas López, M. A. (2012). Educación a distancia: nueva modalidad, nuevos alumnos. Perfiles de alumnos de Psicología en México. Perfiles Educativos, 34(136). Recuperado de http:// www.redalyc.org/articulo.oa?id=13223068008

Pastor Angulo, M. (octubre-diciembre, 2005). La educación superior a distancia en el nuevo contexto tecnológico del Siglo XXI Revista de la Educación Superior, 34(136). Recuperado de http:// publicaciones.anuies.mx/pdfs/revista/ Revista136_S2A4ES.pdf

Peñalosa Castro, E. A., Landa Durán, P. y Castañeda Figueiras, S. (abril-julio 2010). La pericia de los estudiantes como diferenciador del desempeño en un curso en línea. Revista Mexicana de Investigación Educativa, 15(45). Recuperado de http:// www.comie.org.mx/documentos/rmie/v15/ n045/pdf/ART45006.pdf

Peñalosa Castro, E. A. y Castañeda Figueiras, S. (enero-marzo 2008). Generación de conocimiento en la educación en línea: un modelo para el fomento de aprendizaje activo y autorregulado. Revista Mexicana de Investigación Educativa, 13(36). Recuperado de http://www.scielo.org.mx/ scielo.php?script $=$ sci_arttext\&pid $=\mathrm{S} 1405$ 66662008000100011

Ramírez Romero, J. L. (2001). Educación y computadoras: una aproximación al estado actual de su investigación en México. Revista Mexicana de Investigación Educativa, 6(11).

$-136-$

Píxel-Bit. Revista de Medios y Educación. No 52 Enero 2018. ISSN: 1133-8482. e-ISSN: 2171-7966. doi: http://dx.doi.org/10.12795/pixelbit.2018.i52.09 
Recuperado de http://www.redalyc.org/ articulo.oa? id=14001108

Reyes Angona, S., Fernández Cárdenas, M. y Martínez Martínez, R. (2013). Comunidades de blogs para la escritura académica en la enseñanza superior. Un caso de innovación educativa en México. Revista Mexicana de Investigación Educativa, 18(57). Recuperado de http://www.redalyc.org/pdf/140/ 14025774009.pdf

Salinas Amescua, B., Huerta Alva, M. G., Porras Hernández, L. H., Amador Pérez, S. E. y Ramos Rodríguez, J. M. (enero-marzo, 2006). Uso significativo de la tecnología en la educación de adultos en el medio rural: Resultados de la aplicación piloto de un modelo. Revista Mexicana de Investigación Educativa, 11(28). Recuperado de http:// w w w. s c i e 1 o. o r g . $\mathrm{m} \mathrm{x} \mathrm{/}$ scielo.php?script $=$ sci_arttext\&pid $=\mathrm{S} 1665$ 109X2012000200004

Torreblanca Navarro, O. y Rojas Drummond, S. (2010). Mediación tecnológica para el desarrollo de habilidades de observación en estudiantes de Psicología: un enfoque socioconstructivista. Perfiles Educativos, 32(127). Recuperado de http:// www.redalyc.org/articulo.oa?id=13211845004

Torres Velandia, S. A., Barona Ríos, C. y García Ponce de León, O. (2010). Infraestructura tecnológica y apropiación de las TIC en la Universidad Autónoma del Estado de Morelos. Estudio de caso. Perfiles Educativos, 32(127). Recuperado de http:// www.redalyc.org/articulo.oa?id=13211845006

Torres Velandia, S. A. y Jaimes Cruz, K. (2015). Producción de conocimiento mediado por TIC: cuerpos académicos de tres universidades Públicas estatales de México. Revista Electrónica Sinéctica (44). Recuperado de http://www.redalyc.org/ articulo.oa?id $=99832951002$
Urzúa Hernández, M. C. y López Olivas, M. (julio-septiembre 2010). Evaluación de tres intervenciones instruccionales para la formación de una competencia técnica profesional en Química Farmacéutica Biológica. Revista Mexicana de Investigación Educativa, 15(46). Recuperada de http://www.scielo.org.mx/ scielo.php?script $=$ sci_arttext\&pid $=\mathrm{S} 1405$ 66662010000300010

Vidal Puga, M. P. (2006). Investigación de las TIC en la educación. Revista Latinoamericana de Tecnología Educativa, 5(2). Recuperado de file:///C:/Users/ ADRIANA\%20RANGEL/Downloads/ $\begin{array}{llllllllllllllllll} & \mathrm{i} & \mathrm{a} & \mathrm{l} & \mathrm{n} & \mathrm{e} & \mathrm{t} & -\end{array}$ InvestigacionDeLasTICEnLaEducacion2229253.pdf

Fecha de recepción: 31-07-2017

Fecha de evaluación: 10-04-2017

Fecha de aceptación: 14-06-2017

$-137-$

Píxel-Bit. Revista de Medios y Educación. No 52 Enero 2018. ISSN: 1133-8482. e-ISSN: 2171-7966. doi: http://dx.doi.org/10.12795/pixelbit.2018.i52.09 